\title{
Factores de Riesgo Asociados a Sepsis en el Paciente Pediátrico Sometido a Cateterismo Venoso Central (CVC)
}

Risk Factors Associated with in the Pediatric Patients Undergoing

to Central Venous Catheterization (CVC)

\author{
Scheybi Teresa Miralda Méndez* (i) \\ *Intensivista Pediatra,Cuidados Intensivos Pediátricos, Hospital Escuela.
}

\begin{abstract}
RESUMEN
Objetivo.- Identificar los factores de riesgo asociados a sepsis en pacientes pediátricos sometidos a Cateterismo Venoso Central. (CVC) Material y Métodos.- Estudio prospectivo que incluyó 54 pacientes pediátricos mayores de 28 días, ingresados y sometidos a Cateterismo Venoso Central en la Unidad de Cuidados Intensivos Pediátricos y Sala de Emergencias del Hospital Escuela en el período de Julio 2012junio 2013.
\end{abstract}

Resultados.- Fue más frecuente el sexo femenino con proporción 2.2:1.0, la media general fue 92.33 meses \pm 70.7 , predominando $>12$ años $(33.3 \%$; $18 / 54)$ y $5-12$ años $(22.2 \%$; 12/54). La tasa de sepsis fue 4.5/1000 días (19/54, 35.1\%). Instalar el CVC en quirófano fue factor protector de riesgo de sepsis $(p<0.05$; OR 6.97, IC95\%: 1.49-35.9), el número de accesos intravenosos fue mayor entre los fallecidos $(p<0.05)$; así también se asociaron con Infección Asociada a Atención en Salud $(p<0.05)$ con $64.8 \%$ de casos (35/54); a mayor número de horas mayor riesgo de mortalidad $(p<0.05)$, estancia intrahospitalaria total $(p=0.045)$ y en la Unidad de Cuidados Intensivos Pediátricos $(\mathrm{UCIP})(p<0.05)$. La tasa de mortalidad entre pacientes sometidos a CVC fue $9.25 \%$ (5/54), la media del tiempo de supervivencia con CVC fue 328.4 horas ( \pm 15.1 ; IC95\%: 298.7-358.04, Mediana: 336 horas); y Media de supervivencia en días fue 107.572 ( \pm 7.3 ; IC95\%: 92.2-121.9, Mediana 120 días). El cumplimiento de requisitos de asepsia/antisepsia durante el procedimiento fue protector de sepsis $(p<0.05$; OR 6.97, IC95\%: 1.49-35.9), con 88.9\% (48/54) procedimientos realizados con falta o deficiencia de materiales.

Correspondencia:

Scheybi Teresa Miralda Méndez

Cuidados Intensivos Pediátricos, Hospital Escuela

Tegucigalpa, Honduras

dra_scheybi@yahoo.com

$+50431824966$
Conclusión.- La mayor parte de casos son prevenibles, la falta de equipamiento para realizar el procedimiento en condiciones de asepsia es el factor predominante asociado a riesgo de sepsis/mortalidad.

Palabras claves.- Cateterismo Venoso Central; Sepsis; Cuidados críticos; Infección Hospitalaria

\section{ABSTRACT}

Objetive.- To identify the risk factors associated with sepsis in pediatric patients undergoing Central Venous Catheterism (CVC).

Material and Methods.- Prospective study that included 54 pediatric patients older than 28 days, admitted and undergoing Central Venous Catheterization at the Pediatric Intensive Care Unit and Emergency Room at the Hospital School in the period from July 2012 to June 2013.

Results. - The female sex was more frequent in a ratio of 2.2: 1.0, the mean group age was 92.33 months \pm 70.7 , predominantly $>12$ years $(33.3 \%$; $18 / 54)$ and $5-12$ years $(22.2 \%$; $12 / 54)$. The sepsis rate found was $4.5 / 1000$ days (19/54, $35.1 \%)$. Carrying out the procedure in the operating room was a protective factor for sepsis risk ( $p<0.05$; OR 6.97, 95\% Cl: 1.49-35.9), the number of intravenous accesses was higher among those who died $(p<0.05)$; thus they were also associated with nosocomial infection (IN) (p $<0.05)$ with $64.8 \%$ of cases $(35 / 54)$; the greater the number of hours, the greater the risk of mortality $(p<0.05)$ and total hospital stay $(p=0.045)$, and at the Pediatric Intensive Care Unit (PICU) $(p<0.05)$. The mortality rate among patients undergoing CVC was $9.25 \%$ (5/54), the mean survival time with CVC was 328.4 hours $( \pm$ 15.1; 95\% Cl: 298.7-358.04, Median: 336 hours); and Mean survival in days was 107,572 ( \pm 7.3; 95\% Cl: 92.2-121.9, Median 120 days). Compliance with aseptic/antiseptic requirements during the procedure was protective of sepsis $(p<0.05$; OR $6.97,95 \% \mathrm{Cl}$ : $1.49-35.9)$, with $88.9 \%$ (48/54) procedures performed with lack or deficiency of materials. 
Conclusion.- Most cases are preventable, with the lack of equipment to perform the procedure under aseptic conditions the most important factor associated with the risk of sepsis/mortality.

Key Words.- Catheterization, Central Venous; Sepsis; Critical care; Cross Infection

\section{Introducción}

La sepsis asociada a CVC se define como la que se presenta 48 horas después de la colocación del CVC en un paciente previamente no infectado en la UCIP, reportán-dose como casos por 1000 días de cateterismo (1-5). La sepsis es una de las más frecuentes complicaciones graves asociadas al uso de CVC, con mayor riesgo de morbilidad y mortalidad $(6,7)$. Además de ser la complicación más frecuente de la inserción de accesos vasculares centrales, la sepsis asociada a su uso es la que más aumenta por hospitalizaciones prolongadas y el uso de antibióticos de amplio espectro (8). EI CVC provoca disrupción de la integridad de la piel, haciendo posible la infección por bacterias y/o hongos, que se diseminan al torrente sanguíneo, llegando a producir disfunción de órganos, y en consecuencia la muerte $(1,2,5,9)$. La etiología más frecuente son estafilococos coagulasa negativo $(40 \%)$ identificándose en $>50 \%$ de todos los cultivos, seguido por bacilos aerobios gram-negativos $(25 \%)$, Enterococos sp (11-15\%), Candida $s p$ $(6 \%)$, Staphylococcus aureus y raramente micobacterias no tuberculosas $(5,9,10)$. El procedimiento de colocación del Catéter Venoso Central (CVC) es parte integral de la atención al paciente en la Unidad de Cuidados Intensivos Pediátricos (UCIP), proporcionando información hemodinámica, acceso vascular, administración de fármacos y toma de muestras $(1,5,6,10-12)$, extendiéndose su uso dada su utilidad en nutrición parenteral, quimioterapia y manejo por infección localizada; sin embargo la colocación de CVC implica riesgo variable de infección (5-50\%) que puede originarse en el punto de salida del catéter, el túnel o del paquete, la tasa dependerá de factores como el tipo de aparato, la edad del paciente y la condición subyacente, así como el sitio de colocación; la tasa hospitalaria es variable y oscila entre 1.7-4.3/1000 días de catéter $(5,6)$. El diagnóstico específico depende de los recursos laboratoriales disponibles modernos, de alto costo y difícil realización, ya que la infección hemató-gena puede originarse de focos no documentados previamente; clínicamente puede haber eritema local, induración, sensibilidad dolorosa, fluctua-ción y secreción purulenta o con mal olor; depósitos de trombos y fibrina sobre el catéter (5). La evidencia disponible sobre el tratamiento de la infección hematógena asociada a CVC es escasa, la mayor parte deriva de estudios en adultos, series clínicas, guías y algunos estudios de caso-control con bajo nivel de evidencia. La tasa de mortalidad en el caso de infecciones asociadas a infección hematógena por línea central es aproximadamente del $18 \%$ en Estados Unidos de América (EUA), prolongando la hospitalización en promedio siete días y aumentando los costos de forma importante $(1,2,5)$. Otras complicaciones asociadas a la colocación de CVC son hemorragia, punción arterial, oclusión, edema de la extremidad, taponamiento cardíaco, falla de colocación, hidrotórax, hemotórax, neumotórax, infección diseminada con émbolo en la retina, piel, hueso, corazón y órganos viscerales (pulmón, hígado, bazo y riñones), así como disfunción de sistemas de órganos debido a la deposición de complejos inmunes (ejemplo: nefritis) e infección hematógena (5,13). La infección asociada a CVC está relacionada con mortalidad reportados hasta en un $20 \%$ y son la causa del $90 \%$ de las infecciones nosocomiales bacterémicas, estimándose que prolonga la hospitalización siete días y aumenta los costos de forma importante $(1,2,5)$, sin embargo la casuística debe ser estudiada y reportada en cada institución. En Honduras se dispone de algunas publicaciones sobre sepsis neonatal y pediátrica en forma de resumen bibliográfico, guías clínicas y dos estudios clínicos y/o epidemiológicos sobre sepsis en población pediátrica hasta la fecha $(14,15)$, sin datos disponibles sobre el paciente sometido a CVC ni factores asociados a sepsis. En consecuencia, el objetivo de este estudio fue identificar los factores de riesgo asociados a sepsis en pacientes pediátricos sometidos a Cateterismo Venoso Central en la Emergencia/UCIP del Hospital Escuela, con el propósito de generar información de base y de impacto sobre las estrategias a seguir para la disminución de los factores de riesgo. 


\section{Material y Métodos}

Estudio prospectivo que incluyó 54 pacientes pediátricos mayores de 28 días, ingresados y sometidos a CVC en la Sala de Emergencias /UCIP del Hospital Escuela en el período de Julio 2012 a Junio 2013 y que ingresaron sin proceso infeccioso documentado antes de la colocación del CVC. En todos los casos se obtuvo consentimiento firmado previamente informado de padres/responsables, así como se obtuvo aprobación del Comité de Bioética de la Facultad de Ciencias Médicas, fueron excluidos los casos cateterizados en otras salas o fuera del hospital, incluyéndose el total de casos cateterizados en el período en la UCIP/Sala de Emergencias de Pediatría del HEU. La información se recopiló de expedientes clínicos, aplicándose formulario elaborado para este estudio, compuesto por apartados clínicos y evolutivos que incluyen datos generales, factores médicos asociados, características del catéter instalado, clasificación de condiciones de asepsia y complicaciones no infecciosas, número de días intrahospitalarios totales y en UCIP, complementándose con información laboratorial consignada en el expediente clínico, así como diagnósticos de egreso. Se aplicaron las siguientes definiciones de términos:

\section{Sepsis Clínica}

a. Paciente con un patógeno reconocido (ejemplo: Staphylococcus aureus, Pseudomona aeuroginosa) aislado de uno o más hemocultivos, y el patógeno cultivado en sangre no se relaciona con infección en otro sitio.

b. Paciente con al menos un signo o síntoma de infección sistémica (ejemplo: fiebre, escalofríos, o hipotensión), y al menos un cultivo sanguíneo positivo de flora de piel frecuente en el contexto del paciente cateterizado, y el médico instituye tratamiento antibiótico apropiado.

c. Paciente con edad menor de un año que tiene al menos un signo o síntoma o infección sistémica (ejemplo: fiebre, escalofríos, hipotensión, apnea, bradicardia) y al menos un hemocultivo positivo por flora común de piel en el contexto de colocación de catéter, y con médico que instituye tratamiento antimicrobiano apropiado.

2. Tasa de infección: Número de casos/días de cateterismo $\times 1000$

3. Tasa de mortalidad: Fallecimiento/ingresos totales $\times 1000$

Para el procesamiento de información, esta fue digitada en una base de datos electrónica generada con el programa estadístico IBM-SPSS 21.0 (IBM-SPSS, Chicago, IL, EUA, 2012). Con el mismo programa se generaron listados, tablas de frecuencia, tablas de dos variables y comparaciones entre grupos o variables, y se definió como independencia el valor de $p<0.05$. Se realizó comparación de variables categóricas determinando el valor de $\chi^{2}$; así como la determinación de Odds-Ratio (OR) e intervalos de confianza del 95\% (IC95\%:). Se determinó el valor de $r$ para identificar correlación entre variables utilizando el método de Pearson, para variables continuas o discretas, y Spearman para categorías (SPSS 21.0, IBM-SPSS Inc., 2012) con $99 \%$ de confianza, con el mismo programa se calculó el tiempo de supervivencia con la técnica de Kaplan-Meier (K-M).

\section{Resultados}

Se encontró que en los pacientes sometidos a CVC el sexo más frecuente fue el femenino respecto al masculino con una relación de 2.2:1.0 respectivamente. La distribución de la media para la edad del grupo estudiado fue 92.33 meses \pm 70.7 1DE; Rango: 4-204; predominando el grupo de mayores de 12 años (33.3\%; 18/54), seguido por el grupo de 5-12 años $(22.2 \% ; 12 / 54)$ (Tabla 1 )

La tasa de sepsis/bacteremia encontrada fue $4.5 / 1000$ días. La mayor parte de los casos (43/54, 79.6\%) estuvo ingresado en la sección de Críticos de la Sala de Emergencias, no obstante, el $40.7 \%$ de ellos fue sometido a CVC antes de su admisión en UCIP (Tabla 1). Fueron sometidos a transfusión de productos sanguíneos 18/54 (33.3\%) en la sala previa a UCIP, principalmente por anemia severa, aunque no fue un factor de significancia estadística (Tabla 1). 
Tabla 1. Características demográficas y clínicas previas al ingreso a UCIPI

Emergencia del paciente sometidos a CVC. Hospital Escuela,

Julio 2012-Junio 2013 ( $n=54)$

\begin{tabular}{|c|c|c|}
\hline Caracteristica Demográficas & Frecuencia & Porcentaje \\
\hline \multicolumn{3}{|l|}{ Edad } \\
\hline$<1$ año & 5 & $9.3 \%$ \\
\hline 1-2años & 11 & $20.4 \%$ \\
\hline 2.5 años & 8 & $14.8 \%$ \\
\hline $5-12$ años & 12 & $22.2 \%$ \\
\hline$>12$ años & 18 & $33.3 \%$ \\
\hline \multicolumn{3}{|l|}{ Sexo } \\
\hline Masculino & 37 & $68.5 \%$ \\
\hline Femenino & 17 & $31.5 \%$ \\
\hline \multicolumn{3}{|l|}{ Caracteristicas y Evolución preingreso } \\
\hline \multicolumn{3}{|l|}{$\begin{array}{c}\text { Horas preingreso a UCIP } \\
\text { M: 30.1; DS: 23.3; R: } 1 \text {-168 }\end{array}$} \\
\hline$<24$ horas & 10 & $18.5 \%$ \\
\hline 24-48 horas & 43 & $79.6 \%$ \\
\hline 78 horas & 1 & $1.9 \%$ \\
\hline \multicolumn{3}{|l|}{ Tipo de catéter colocado en sala previa } \\
\hline Periférico & 29 & $53.7 \%$ \\
\hline Perif́érico y central & 18 & $33.3 \%$ \\
\hline Central & 4 & $7.4 \%$ \\
\hline Aiterial & 3 & $5.6 \%$ \\
\hline \multicolumn{3}{|l|}{ Transfusión en sala previa } \\
\hline $\mathrm{Si}$ & 18 & $33.3 \%$ \\
\hline
\end{tabular}

Fuente: Instrumento

La comorbilidad se identificó en 19/54 (35.2\%), presentaban al ingreso algún grado de desnutrición 20/54 (37.04\%); entre las indicaciones de ingreso se encontraron paciente crítico postquirúrgico, fue la más frecuente seguida de falla ventilatoria y politraumatismo en orden de frecuencia; el diagnóstico de ingreso más frecuente falla ventilatoria 46/54 (85.2\%) (Tabla 2).
Tabla 2. Características Clínicas al ingreso en UCIP/Emergencia del paciente sometidos a CVC. Hospital Escuela, Julio 2012-Junio 2013 ( $n=54)$

\begin{tabular}{|c|c|c|}
\hline Característica & Frecuencia & Porcentaje \\
\hline Comorbilidad & & \\
\hline Postquirúrgico & 16 & $29.6 \%$ \\
\hline Neutropenia secundaria & 2 & $3.7 \%$ \\
\hline Sindrome genético & 1 & $1.9 \%$ \\
\hline Estado nutricional & & \\
\hline Eutróico & 34 & $63.0 \%$ \\
\hline DPC aguda I & 7 & $13.0 \%$ \\
\hline DPC gaguda II & 11 & $20.4 \%$ \\
\hline DPC aguda III & 1 & $1.9 \%$ \\
\hline DPC aguda II crónica II & 1 & $1.9 \%$ \\
\hline Diagnósticos de Ingreso a UCIP & & \\
\hline Postoperado & 16 & $29.6 \%$ \\
\hline Falla ventilatoria & 13 & $24.1 \%$ \\
\hline Politraumatismo & 7 & $13.0 \%$ \\
\hline TEC abiertotCC Severa & 5 & $9.3 \%$ \\
\hline Sindrome Guillián Barré & 3 & $5.6 \%$ \\
\hline TEC cerrado+CC Severa & 3 & $5.6 \%$ \\
\hline Estatus convulsivo & 2 & $3.7 \%$ \\
\hline Quemadura por electricidad 100\% SCQ & 1 & $1.9 \%$ \\
\hline Leucemia Linfoćtica Aguda (LLA) & 1 & $1.9 \%$ \\
\hline Fibrosis quística & 1 & $1.9 \%$ \\
\hline Diabetes insípida nefrogénica & 1 & $1.9 \%$ \\
\hline Insuficiencia Renal Aguda (IRA) & 1 & $1.9 \%$ \\
\hline
\end{tabular}

Fuente: Instrumento

El uso de bloqueadores $\mathrm{H} 2$, la nutrición parenteral y hemoderivados no se asoció con riesgo de complicaciones infecciosas en pacientes con CVC, ni las respuestas terapéuticas, ni riesgo de mortalidad $(p>0.05)$. Fueron sometidos a nutrición parenteral $5 / 54$ $(9.3 \%)$ (Tabla 3$) ;$ y no se encontró relación con infección asociada a atención en salud $(p=0.646$, rango 0.259), ni con sepsis, DIH totales $(p=0.047)$ o días en UCIP (R Pearson: 0.274), y se asoció a incapacidad para alimentarse y condición de egreso $(p=0.021, R: 0.331)$, encontrándose probable asociación estadística fronteriza entre este y prolongación de estancia intrahospitalaria total $(p=0.047$; Pearson $r$ : 0.259, P Pearson: 0.059), asociación que desaparece al tratar de relacionarla con DIH en la UCIP $(p>0.05)$.

El número de accesos intravenosos al momento del ingreso a UCIP se correlacionó con infección asociada a atención en salud ( $p=0.001 ; R$ : 0.530; sig. 0.000) y con la disminución de la respuesta terapéutica a la antibióticoterapia $(p=0.000$; OR: celda en $0, r: 0.363)(p=0.001)$. La tasa de infección intrahospitalaria fue $35 / 54$ casos $(64.81 \%)$, de Neumonía Nosocomial 20/54 (37.0\%) y sepsis 19/54 (35.1\%) (Tabla 3 ). 
Tabla 3. Factores médicos asociados a riesgo de sepsis en pacientes sometidos a CVC en UCIP/Emergencia. Hospital Escuela, Julio 2012-Junio 2013 ( $n=54$ )

\begin{tabular}{|c|c|c|c|}
\hline Factores & Sepsis & No Sepsis & $p$ \\
\hline Nutricón parenteral & & & 0.264 \\
\hline Si & 4 & 1 & \\
\hline No & 31 & 18 & \\
\hline Número de accesos intravenosos al ingreso & & & 0.208 \\
\hline 1 & 14 & 4 & \\
\hline 2 & 16 & 9 & \\
\hline 3 & 5 & 6 & \\
\hline Trannsusiones & & & 0.98 \\
\hline Si & 13 & 7 & \\
\hline No & 22 & 12 & \\
\hline $\begin{array}{c}\text { Infección asociada a atención en salud } \\
\text { después de ingreso a UClP }\end{array}$ & 35 & 19 & \\
\hline Diagnósico neumonía nosocomial en UCIP & 4 & 16 & 0.13 \\
\hline Diagnósicico de sepsis en UCIP & 19 & & \\
\hline $\begin{array}{c}\text { Númmero de accesos intravenosos al } \\
\text { momento del diagnóstico de sepsis }\end{array}$ & & & 0.001 \\
\hline 0 & 16 & 19 & \\
\hline 1 & 11 & 0 & \\
\hline 2 & 6 & 0 & \\
\hline 3 & 2 & 0 & \\
\hline \multicolumn{3}{|c|}{ Fuente: Instrumento }
\end{tabular}

La colocación del CVC por médico especialista intensivista o médico residente de cuidados intensivos fue de $36 / 54(66.67 \%)$, el resto lo colocó cirujanos 9/54 (16.67\%) o residentes III año de pediatría $(8 / 54,14.81 \%)$ (Tabla 4). La persona que colocó el CVC y que se relacionó con menor porcentaje de casos de sepsis fue el cirujano comparado con el resto del personal $(p<0.05 ; r-0.398)$ (Tabla 4). De acuerdo con el personal que coloca el CVC y riesgo de infección asociada a atención en salud diagnosticada en UCIP, se encontró que la colocación del CVC por parte de Anestesiólogo/Cirujano es 7 veces protector de infección asociada a atención en salud ( $p<0.05$; OR 6.97, IC95\%: 1.49-35.9).
Tabla 4. Características de instalación de CVC en pacientes atendidos la Unidad de Cuidados Intensivos Pediátricos (UCIP)/Emergencia. Hospital Escuela, Julio 2012-Junio $2013(n=54)$

\begin{tabular}{|c|c|c|c|}
\hline Características ( $n=54$ ) & Sepsis & No Sepsis & $p$ \\
\hline Catéter 1 & & & 0.001 \\
\hline \multicolumn{4}{|l|}{ Lugar/Sala donde se coloca catéter } \\
\hline Emergencia & 20 & 7 & \\
\hline UCIP & 12 & 2 & \\
\hline Quirófano & 3 & 10 & \\
\hline Tamaño/lumen adecuado para el paciente & & & 0.704 \\
\hline $\mathrm{Si}$ & 28 & 16 & \\
\hline No & 7 & 3 & \\
\hline Catéteres instalados simultáneamente & & & 0.342 \\
\hline $\mathrm{Si}$ & 4 & 4 & \\
\hline No & 31 & 15 & \\
\hline Sitio de inserción de catéter & & & 0.213 \\
\hline Subclavio izquierdo & 8 & 1 & \\
\hline Subclavio derecho & 3 & 3 & \\
\hline Femoral izquierdo & 14 & 8 & \\
\hline Femoral derecho & 9 & 5 & \\
\hline Yugular derecha & 0 & 2 & \\
\hline Yugular izquierda & 1 & 0 & \\
\hline Persona que coloca catéter & & & 0.026 \\
\hline Pediatra & 1 & 0 & \\
\hline Intensivista & 3 & 0 & \\
\hline Residente III & 6 & 2 & \\
\hline Residente Cuidados Intensivos IV & 14 & 2 & \\
\hline Residente Cuidados Intensivos V & 7 & 6 & \\
\hline Cirujano & 2 & 7 & \\
\hline Anestesiólogo & 2 & 2 & \\
\hline Turno en que se coloca catéter & & & 0.078 \\
\hline A & 11 & 12 & \\
\hline $\mathrm{B}$ & 16 & 5 & \\
\hline$C$ & 8 & 2 & \\
\hline Tiempo en minutos en que se coloca catéter: & & & 0.857 \\
\hline 10-15 minutos & 14 & 14 & \\
\hline $15-30$ minutos & 11 & 18 & \\
\hline$>30$ minutos & 1 & 3 & \\
\hline Tiempo en horas en que está colocado el & & & 0.000 \\
\hline \multicolumn{4}{|l|}{ catéter: } \\
\hline$<10$ horas & 0 & 2 & \\
\hline 10-24 horas & 3 & 4 & \\
\hline 24-48 horas & 1 & 4 & \\
\hline $48-120$ horas & 5 & 7 & \\
\hline$>120$ horas & 26 & 2 & \\
\hline
\end{tabular}

El sitio de colocación del CVC no se relacionó con riesgo de infección asociada a atención en salud, sepsis, estancia intrahospitalaria, condición de egreso $(p>0.05)$. La media en tiempo de colocación de CVC fue 20.1 minutos (DE \pm 9.31, $\mathrm{R}: 10-50$ ), la mayor frecuencia fue entre 15-30 minutos 29/54 (53.7\%) (Tabla 4). No se asoció el tiempo en minutos en que se coloca catéter después de ingreso en UCIP ( $p>0.05)$. La media del tiempo en horas que permaneció colocado el CVC fue 144.7 horas (DE \pm 94.3 R: 1-360), 
predominando el grupo con más de 120 horas en $28 / 54$ casos $(51.9 \%$ ) (Tabla 4$)$. Se encontró asociación entre horas de CVC y condición de egreso $(p=0.000)$, así como con prolongación de la estancia intrahospitalaria en UCIP en días $(p=0.039 ; R: 0.532)$, y con días de estancia intrahospitalaria totales $(p=0.045 ; R$ : 0.586$)$. No se relacionó con sepsis o Neumonía Nosocomial $(p>0.05)$. No se relacionó con riesgo de transfusión ni infección asociada a atención en salud. Se correlacionó de forma moderada con respuesta terapéutica ( $R$ Pearson: 0.522), e infección asociada a atención en salud (R: 0.540). Se obtuvo buena respuesta al tratamiento empírico en $31.5 \%$ (17/54), la necesidad de cambio de antibiótico empírico inicial se relaciona con la estancia intrahospitalaria $(p=0.000$; ORIC95\%: $0.02<0.10<0.48)$ R: 0.536$)$, pero no con sepsis ni condición de egreso $(p>0.05)$. Entre la calificación de condiciones de asepsia, en los catéteres colocados en la Emergencia/UCIP en la mayor parte de los casos se consignó falta de materiales o deficiencia de alguno de éstos o de las prácticas $(48 / 54,88.9 \%)$, la mayor parte de casos presentó al menos una de estas fallas con 35/54 (64.8\%), (Tabla 5). Respecto al tipo de complicación no infecciosa la más frecuente fue retención de guía de CVC y extravasación, no encontrándose diferencia estadística entre grupos $(p<0.05)($ Tabla 5$)$; las punciones fallidas se presentaron en $35 / 56$ pacientes $(76.09 \%)$ sin diferencia entre grupos $(p=0.916)$; en la mayor parte de casos se realizó más de una manipulación al día; aunque esto no alcanzó significancia estadística al intentar relacionarla con riesgo de sepsis $(p=0.055)$. El número de accesos intravenosos fue $>1$ en la mayoría de los pacientes con sepsis, con diferencia estadística entre grupos ( $p=0.000$, RR. 2.19; IC95\%: 1.5-3.1) (Tabla 5).
Tabla 5. Variables epidemiológicas sobre factores de riesgo asociadas a sepsis en pacientes sometidos a CVC en UCIP/Emergencia. Hospital Escuela, Julio 2012-Junio 2013 ( $n=54)$

\begin{tabular}{|c|c|c|c|}
\hline Evolución (n=54) & Sepsis & No sepsis & $p$ \\
\hline Callificación de condiciones de asepsia & & & 0.96 \\
\hline Deficiencia de suminisitro & 18 & 5 & \\
\hline No realiza Técnica de asepsia & 0 & 1 & \\
\hline No realiza Lavado de manos & 0 & 1 & \\
\hline No dissone de Equipo de ciruggía menor & 1 & 0 & \\
\hline No uso de ropa estéril & 28 & 18 & \\
\hline No uso de catéter a adecuado & 6 & 3 & \\
\hline No uso de campos estériles & 11 & 3 & \\
\hline No uss de bata & 2 & 0 & \\
\hline Tipo de complicación no infecciosa & & & 0.701 \\
\hline \multicolumn{4}{|l|}{ presentada } \\
\hline Retención de guía de CVC & 3 & 1 & \\
\hline Extravasación & 3 & 0 & \\
\hline Neumotórax & 2 & 0 & \\
\hline Hemotórax & 0 & 1 & \\
\hline Ubicación inadecuada & 1 & 0 & \\
\hline Número de punciones fallididas & & & 0.916 \\
\hline 0 & 11 & 8 & \\
\hline 1.2 & 7 & 2 & \\
\hline $3-4$ & 14 & 7 & \\
\hline 5.6 & 2 & 2 & \\
\hline $7 \cdot 10$ & 1 & 0 & \\
\hline \multicolumn{4}{|l|}{ Método de Identificación del agente } \\
\hline Hemocultivo & 2 & 0 & \\
\hline Secreción & 2 & 0 & \\
\hline Punta de catéter $A$ & 1 & 0 & \\
\hline spirado TET & 1 & 0 & \\
\hline Número de accesos intravenosos al & & & 0.001 \\
\hline \multicolumn{4}{|l|}{ Momento del diagnóstico de sepsis } \\
\hline 1 & 16 & 19 & \\
\hline 2 & 11 & 0 & \\
\hline 3 & 6 & 0 & \\
\hline Transíusiones & 2 & 0 & \\
\hline Manipulaciones al día & & & 0.055 \\
\hline 2.5 & 4 & 6 & \\
\hline 6.10 & 13 & 9 & \\
\hline 11-17 & 18 & 4 & \\
\hline Condición de egreso & & & 0.813 \\
\hline Vivo & 17 & 32 & \\
\hline Muerto & 2 & 3 & \\
\hline
\end{tabular}


La media de estancia intrahospitalaria total fue 19.7 días (DE \pm 22.41 ; R: 0.7-120) con mayor frecuencia en el grupo de 1-7 días con 29/54 $(53.7 \%)$. La tasa de mortalidad fue $9.25 \%(5 / 54)$. La media de tiempo de supervivencia sometido a CVC fue 328.4 horas ( \pm 15.1 ; IC95\%: 298.7$358.04)$ y mediana de 336 horas. La media de supervivencia en días fue 107.572 ( \pm 7.3 ; IC95\%: 92.2-121.9) con mediana de 120 días.

\section{Discusión}

Debido a que el tiempo de permanencia del cateterismo influye sobre el riesgo de infección, típicamente se utiliza el indicador días-catéter como denominador estándar para reportar tasa de infección, en este estudio la tasa de infección fue 4.5/1000 días, que es superior a lo reportado en Chile con 2.2/1000 días, aunque inferior a lo reportado a nivel centroamericano en el Hospital Nacional de Niños Benjamín Bloom de El Salvador de $10 / 1000$ días, e inferior a la reportada en EUA que es de 6.6/1000 días de catéter.(1, 3-5)

En España y Portugal la mortalidad es de aproximadamente $5.0 \%$, mientras que para Latinoamérica el porcentaje promedio es $13.29 \%$; a nivel centroamericano Honduras reporta la mortalidad general en UCIP más alta con 25\%; encontrándose en este reporte que el aporte a esta tasa de la mortalidad de casos sometidos a CVC fue $9.25 \%$, por debajo de la media nacional, pero muy por encima de Chile, Uruguay, aunque inferior de la reportada para República Dominicana, Guatemala, México, Ecuador y Colombia y EUA $(1,2,5,16)$; no obstante, es de hacer notar que se está comparando una unidad en particular con la media nacional de mortalidad general en UCIP para cada país, y en este estudio se reporta en pacientes con CVC, lo que podría explicar las diferencias encontradas por el autor.

Entre los factores de riesgo por sepsis y mortalidad reportados están uso de catéter arterial, uso de bloqueadores $\mathrm{H} 2$, nutrición parenteral, transfusión con hemoderivados y defectos o síndrome genéticos; así como la proporción de enfermeras y pacientes, número de accesos intravenosos $(11,17,18)$, en los resultados mostrados no se identificó asociación alguna entre estos factores y sepsis ó mortalidad; aunque se encontró asociación entre número de accesos intravenosos y sepsis en el grupo estudiado $(p<0.05)$, esto posiblemente relacionado con el hallazgo de que casi dos tercios de los casos presentaron infección intrahospitalaria y más de un tercio diagnóstico de neumonía nosocomial (Tabla 3), con un tercio en quienes se consignó el diagnóstico de sepsis; la etiología es variable y consiste en gramnegativos aerobios y enterococos con casos raros asociados a micobacterias no tuberculosas (5); en el grupo estudiado se identificó agente únicamente en seis casos, todos gramnegativos y dos casos fueron enterococos para una tasa global de detección de $11.0 \%$.

Las complicaciones infecciosas y no infecciosas asociadas al uso de CVC ocurren con frecuencia en las UCIP $(7,12,19)$; estas incluyen sepsis, infección diseminada con émbolo en la retina, piel, hueso, corazón, y órganos viscerales (pulmón, hígado, bazo y riñones), así como disfunción de sistemas de órganos debido al depósito de complejos inmunes (ejemplo: nefritis);(5) la proporción de casos encontrada por el autor fue de poco más de un quinto de la muestra estudiada, predominando la retención de guía y la extravasación posiblemente relacionadas más con la logística y equipamiento de la unidad que con otros factores.

Respecto a la prevención de infecciones asociadas al CVC, se recomienda el uso de preparaciones cutáneas de gluconato de clorhexidina y barreras estériles máximas durante la inserción, uso de vena yugular subclavia o interna en lugar de la vena femoral, higiene de manos y revisión diaria $(6,20)$. En una revisión llevada a cabo por O'Grady y colaboradores quienes establecen las pautas generales para implementar medidas de impacto en la unidad de cuidados intensivos pediátrica, citando los autores múltiples métodos y estrategias (10); sin embargo, en esta unidad de acuerdo a los hallazgos realizados por el autor algunos de los problemas para implementar dichas medidas son de índole logística, que es la base primaria de todas las medidas 0 estrategias de prevención de morbilidad y mortalidad. 
Uno de los hallazgos de este estudio y relacionados con la inferencia anteriormente expuesta, es que entre los pacientes cateterizados con CVC, se identificó como factor protector de sepsis la colocación del mismo en quirófano por el cirujano o el anestesiólogo, incluyendo protección de riesgo por infección asociada a atención en salud ( $\mathrm{p}<0.05$; OR: 6.97; IC95\%: 1.49-35.9); el quirófano normalmente tiene todo el equipamiento y condiciones para la mejor asepsia posible en la institución, lo que remarca el papel de la misma en la prevención de sepsis o infección asociada a atención en salud en este grupo de pacientes; de hecho en la calificación de condiciones de asepsia en los procedimientos realizados en la Emergencia / UCIP se identificó la falta de materiales o deficien-cia de alguno de éstos en más de cuatro quintas partes del total de procedimientos en esas salas; también se identificó al menos una falla en la técnica de asepsia en casi dos tercios de todos los procedimientos realizados en las mismas (Tabla 4).

Algunos factores relacionados por la literatura asociados a riesgo de complicación infecciosa o no infecciosa son el tiempo de colocación, número de punciones fallidas, número de manipulaciones, sitio de colocación, tiempo de permanencia del CVC $(9,10,13,21-23)$; en este estudio no se identificó a ninguno de estos factores como relacionadas con riesgo de sepsis, infección asociada a atención en salud ó mortalidad; aunque hay que hacer notar que a mayor tiempo en horas que permanece el CVC en el paciente, hay mayor riesgo encontrándose correlación moderada con el evento de muerte del paciente ( $p=0.000$; R Spearman: 0.520$)$, así como a estancia hospitalaria total prolongada $(p=0.039)$.

Este reporte representa el inicio de la línea de investigación sobre Cateterismo Venoso Central en pacientes pediátricos en unidad de cuidados críticos a nivel nacional y hospitalario, por ser prospectivo y delinear las características y evolución clínica, así como los principales factores relacionados con riesgo de sepsis, mortalidad y prolongación de estancia intrahospitalaria propios de nuestro medio. Entre las limitaciones del estudio está el tamaño de la muestra y que debido a falta de recursos laboratoriales no fue posible diagnosticar los casos de bacteremia.
El autor concluye que la tasa de infección es aceptable de acuerdo con el contexto de recursos de la institución; no obstante, la mayor parte de los casos son prevenibles y asociados con falta de equipamiento para realizar el procedimiento en condiciones de asepsia, que se asocia directamente con las fallas encontradas en el procedimiento y que afectó a la mayor parte de los casos, posiblemente esto actúa como confusor para la identificación de otros factores reportados en la literatura.

Por lo que se recomienda suministrar material suficiente y equipo apropiado para realizar el procedimiento y cumplir con las normas requeridas de asepsia y antisepsia tanto de personal médico y paramédico e incrementar la actividad alrededor de conocimientos y prácticas en el personal de enfermería y de jefatura, así como aplicar las medidas de prevención recomendadas con evaluaciones continuas y minimizar la rotación del personal. Disminuir el tiempo de permanencia del CVC e implementar un protocolo de estandarización de manejo del paciente con CVC.

\section{Bibliografía}

1. Miller-Hoover S. Pediatric central line: bundle implementation and outcomes. J Infus Nurs. 2011;34(1):36-48. Epub 2011/01/18.

2. Richardson J, Tjoelker R. Beyond the central line-associated bloodstream infection bundle: the value of the clinical nurse specialist in continuing evidence-based practice changes. Clin Nurse Spec. 2012;26(4):205-11. Epub 2012/06/09.

3. Sexton DJ, Chen LF, Anderson DJ. Current definitions of central line-associated bloodstream infection: is the emperor wearing clothes? Infect Control Hosp Epidemiol. 2010;31(12):1286-9. Epub 2010/10/29.

4. Tokars JI, Klevens RM, Edwards JR, Horan TC. Measurement of the impact of risk adjustment for central line-days on interpretation of central line-associated bloodstream infection rates. Infect Control Hosp Epidemiol. 2007;28(9):1025-9. Epub 2007/10/13. 
5. Smith M, Shah S. Device-related Infections. In: Bergelson J, Shah S, Zaoutis T, editors. Pediatric Infectious Diseases: The Requisites in Pediatrics Chapter 35. Philadelphia, PA: Mosby-Elsevier Inc; 2008. p. 331-5.

6. Pinelli F, Cecero E, Degl'Innocenti D, Selmi V, Giua R, Villa G, et al. Infection of totally implantable venous access devices: A review of the literature. The journal of vascular access. 2018;19(3):230-42. Epub 2018/03/08.

7. Litz CN, Tropf JG, Danielson PD, Chandler NM. The idle central venous catheter in the NICU: When should it be removed? Journal of pediatric surgery. 2018;53(7):1414-6. Epub 2017/12/05.

8. Woeltje KF, Butler AM, Goris AJ, Tutlam NT, Doherty JA, Westover MB, et al. Automated surveillance for central line-associated bloodstream infection in intensive care units. Infect Control Hosp Epidemiol. 2008;29(9):842-6. Epub 2008/08/21.

9. Simon A, Fleischhack G, Hasan C, Bode U, Engelhart S, Kramer MH. Surveillance for nosocomial and central line-related infections among pediatric hematologyoncology patients. Infect Control Hosp Epidemiol. 2000;21(9):592-6. Epub 2000/09/23.

10. O'Grady NP, Alexander M, Burns LA, Dellinger EP, Garland J, Heard SO, et al. Guidelines for the prevention of intravascular catheter-related infections. Clin Infect Dis. 2011;52(9):e162-93. Epub 2011/04/05.

11. Advani S, Reich NG, Sengupta A, Gosey L, Milstone AM. Central line-associated bloodstream infection in hospitalized children with peripherally inserted central venous catheters: extending risk analyses outside the intensive care unit. Clin Infect Dis. 2011;52(9):1108-15. Epub 2011/04/02.

12. Wen J, Yu Q, Chen H, Chen N, Huang S, Cai W. Peripherally inserted central venous catheter-associated complications exert negative effects on body weight gain in neonatal intensive care units. Asia Pacific journal of clinical nutrition. 2017;26(1):1-5. Epub 2017/01/05.
13.Díaz M, Rivera L, Arango M, Rodríguez R. Cateterismo Venoso Central Percutáneo en Neonatos: Preferencias, Indicaciones y Complicaciones. Rev Cubana Pediatr. 2006;78(3):1.14.

14. Cerrato-Molina T, Sánchez C. Caracterización de sepsis grave en pacientes ingresados en la Unidad de Cuidados Intensivos y Emergencia Pediática del Hospital Escuela. Rev Med Post UNAH. 2007;10(1):13, 4.

15.Soto A, Zúñiga C, Borjas G, Flores I, Zelaya $\mathrm{J}$, Maradiaga $\mathrm{J}$, et al. Factores de Riesgo Involucrados en la Sepsis de Lactantes ingresados en el Hospital Escuela durante el mes de Junio de 2008. Rev Fac Cienc Med. 2009;6(1):17-25.

16.Campos-Mino S, Sasbon JS, von Dessauer B. [Pediatric intensive care in Latin America]. Med Intensiva. 2012;36(1):3-10. Epub 2011/09/13. Los cuidados intensivos pediatricos en Latinoamerica.

17.Elward AM, Fraser VJ. Risk factors for nosocomial primary bloodstream infection in pediatric intensive care unit patients: a 2year prospective cohort study. Infect Control Hosp Epidemiol. 2006;27(6):553-60. Epub 2006/06/07.

18.Wylie MC, Graham DA, Potter-Bynoe G, Kleinman ME, Randolph AG, Costello JM, et al. Risk factors for central line-associated bloodstream infection in pediatric intensive care units. Infect Control Hosp Epidemiol. 2010;31(10):1049-56. Epub 2010/08/25.

19.Yamaguchi RS, Noritomi DT, Degaspare NV, Munoz GOC, Porto APM, Costa SF, et al. Peripherally inserted central catheters are associated with lower risk of bloodstream infection compared with central venous catheters in paediatric intensive care patients: a propensity-adjusted analysis. Intensive care medicine. 2017;43(8):1097104. Epub 2017/06/07.

20.Devrim I, Oruc Y, Demirag B, Kara A, Duzgol $M$, Uslu S, et al. Central line bundle for prevention of central line-associated bloodstream infection for totally implantable venous access devices (ports) in pediatric cancer patients. The journal of vascular access. 2018;19(4):358-65. Epub 2018/06/22. 


\section{ARTÍCULO ORIGINAL}

21.Kime T, Mohsini K, Nwankwo MU, Turner B. Central line "attention" is their best prevention. Adv Neonatal Care. 2011;11(4): 242-8; quiz 9-50. Epub 2011/11/30.

22. Calvo M. Infecciones asociadas a catéteres. Revista Chilena de Medicina Intensiva. 2008;23(2):94-103.

23. Rosenthal VD, Maki DG, Rodrigues C, Alvarez-Moreno C, Leblebicioglu H, Sobreyra-Oropeza $\mathrm{M}$, et al. Impact of International Nosocomial Infection Control Consortium (INICC) strategy on central lineassociated bloodstream infection rates in the intensive care units of 15 developing countries. Infect Control Hosp Epidemiol. 2010;31(12):1264-72. Epub 2010/10/30.

Conflicto de interés: Ninguno declarado 